\title{
Simón Bolívar (1783-1830) und die Verfassung der „Bolivarischen Republik Venezuela“ von 1999: eine verfassungsgeschichtliche Bestandsaufnahme ${ }^{1}$
}

\author{
Von Andreas Timmermann, Berlin
}

\section{Einführung}

Die Geburt der neuen, „bolivarischen Bewegung“ ist als pathetische Szene überliefert. Hugo Chávez Frías, Lehrer an der Militärakademie, leistet mit zwei weiteren Offizieren am 17. Dezember 1982 unter einem Baum in Samán Güere, nahe der Stadt Maracay, den feierlichen Eid: „Ich schwöre vor Euch und vor dem Gott meiner Väter, dass mein Arm nicht eher ruht und meine Seele nicht eher rastet, bis ich nicht die Ketten zerbrochen habe, die uns fesseln“ “2. Anlass dieses Eides waren die landesweiten Feierlichkeiten zum 200. Geburtstag Simón Bolívars (1783), des „Befreiers“ von der spanischen Herrschaft, den dieser in Rom im Jahr 1805 geschworen haben will ${ }^{3}$. Auch die von den Verschwörern gegründete politische Zelle in der Armee, der ,Movimiento Bolivariano Revolucionario 200“ (MBR-200), bedient sich des Namens und Andenkens Simón Bolívars. Von nun an begründete Hugo Chávez alle weiteren Schritte auf dem Weg zur Machtergreifung mit dem Staatsdenken und Regierungshandeln des „Befreiers“, ebenso die weiteren Maßnahmen nach seiner Wahl zum Präsidenten der Republik im Jahr 1998: Dies gilt für seinen erfolglosen Putschversuch vom 4. Februar 1992 gegen die Regierung des Präsidenten Carlos Andrés Pérez wie auch für den politischen Neuanfang nach Begnadigung und Haftentlassung (1994). Chávez stellte jener ursprünglich militärischen Gruppierung des MBR-200 eine parteiähnliche Organisation, den „Movimiento Quinta República“ (MVR), an die Seite und trat $1998 \mathrm{zu}$ den Präsidentschaftswahlen an, um eine nunmehr ,friedliche bolivarische

Der Beitrag ist die erweiterte Fassung eines Referats, das der Autor auf der Jahrestagung des Arbeitskreises für Überseeische Verfassungsvergleichung (AÜV) am 10. Juli 2004 in Göttingen gehalten hat.

Richard Gott, In the Shadow of the Liberator. Hugo Chávez and the transformation of Venezuela, London 2000, S. 40; die beiden Mitverschwörer, ebenfalls Dozenten an der Militärakademie von Caracas, waren Felipe Acosta Charles und Jesús Urdaneta Hernández.

3

Zum Eid O. Carlos Stoetzer, Bolívar und Europa (1783-1830), in: Wilhelm Stegmann (Hg.), Simón Bolívar: Persönlichkeit und Wirkung, Berlin 1984, S. 62. 
Revolution“ zu verwirklichen ${ }^{4}$. Nach Maßgabe des Regierungsprogramms besteht die politische Zielsetzung der von ihm angeführten Regierungskoalition ${ }^{5}$ - auch hier unter Berufung auf den Gründermythos der Republik Venezuela - in der Errichtung einer ,bolivarischen Demokratie“" ${ }^{6}$. Die am 15. Dezember 1999 durch ein Referendum angenommene neue Verfassung bezieht sich nicht nur im Titel, sondern auch in der Präambel auf das politische Vermächtnis Simón Bolívars ${ }^{7}$. Wenn es hier noch ganz allgemein um „,das historische Beispiel unseres Befreiers" sowie den Heroismus jener Vorfahren und Vorläufer der Unabhängigkeit geht, benennt Art. 1 der Verfassung die „Lehre Simón Bolívars, des Befreiers“, als die grundlegende staatsrechtliche Quelle für die neue Verfassung ${ }^{8}$. Etwas überspitzt lässt sich zusammenfassen, dass von den obersten Prinzipien der Staatsordnung bis hin zu Einzelprojekten beinahe alles mit dem Namen Simón Bolívar verknüpft wird, ob es sich um die Bildungseinrichtungen (,Escuelas Bolivarianas“) oder um Sozial- und Entwicklungsprogramme („Proyecto Bolívar 2000“) handelt. ${ }^{9}$

Die Frage, ob sich Hugo Chávez in seinem Staatsdenken und -handeln zu Recht oder Unrecht auf Simón Bolívar bezieht, wird je nach der weltanschaulichen Position unterschiedlich beurteilt. Die Kritiker erkennen keinerlei historische Parellelen zur Gründungsgeschichte Venezuelas im allgemeinen und zur Persönlichkeit Bolívars im besonderen. Die Berufung hierauf diene allein einem politischen Kult der Tadition, um die in der Bevölkerung besonders leicht anzusprechenden ,paternalistischen und messianischen Reflexe“ auszunutzen. Dies sei besonders deshalb erfolgversprechend, weil an die beiden in Venezuela am tiefsten verwurzelten Mythen appelliert werde: den militärischen und den Gründer-

Dazu Friedrich Welsch / Nikolaus Werz, Der Wahlsieg und der Regierungsbeginn von Hugo Chávez Frías in Venezuela, Rostock 1999, S. 10; Nicole Monleón, Verfassungsrechtliche Aspekte der Krise in Venezuela, in: Verfassung und Recht in Übersee 37 (2004), S. 61.

„Movimiento Quinta República“ (MVR), „Movimiento al Socialismo” (MAS), „Patria para todos” (PPT), „Partido Comunista” (PC).

6

So der Untertitel des 1. Abschnitts („Construyamos la Democracia Bolivariana“), unter: www. mpd.gov.ve/prog-gob/prog_gob2/indice.htm, S. 3 .

„Constitución de la República Bolivariana de Venezuela”, unter: www.georgetown.edu/pdba/Constitutions/Venezuela/ven1999.html. Der erste Satz der Präambel lautet: „El pueblo de Venezuela, en ejercicio de sus poderes creadores e invocando la protección de Dios, el ejemplo histórico de nuestro Libertador Simón Bolívar y el heroísmo y sacrificio de nuestros antepasados aborígines y de los precursores y forjadores de una patria libre y soberana" (S. 1).

„La República Bolivariana de Venezuela es irrevocablemente libre e independiente y fundamenta su patrimonio moral y sus valores de libertad, igualdad, justicia y paz internacional, en la doctrina de Simón Bolívar, el Libertador” (Art. 1 des 1. Titels der Verfassung: „Principios fundamentales"), in: Verfassung (Anm. 7), S. 1.

Regierungsprogramm (Anm. 6), S. 28 bzw. S. 34 ff. 
mythos ${ }^{10}$. Die Gegenansicht folgt dem historischen Rekurs bei Chávez, insofern sie vermeintliche Gemeinsamkeiten mit Bolívar zu einer Identität von Werdegang und Programmatik überhöht: Sie sieht im Regierungsantritt des Präsidenten Chávez und in der Ausarbeitung der neuen Verfassung den Übergang vom Alten Staat, dem ,ancien régime“ der (Zwei)parteienherrschaft, zu einem neuen, nun demokratischen Staat. Dieser Übergang habe sich nach verschiedenen Revolten: 1989 als unorganisierte Volksbewegung, dem „Caracazo“, und 1992 in zwei militärischen Konspirationen schließlich mit dem Wahlsieg Chávez' 1998 vollzogen. ${ }^{11}$

\section{Schwierigkeiten eines Vergleichs}

Abgesehen von der grundsätzlichen Problematik solcher Vergleiche über einen zweihundertjährigen Zeitraum hinweg, ist die Frage, ob Parallelen zu Simón Bolívar und zur Überwindung des ,antiguo régimen“ der spanischen Monarchie in den Jahren nach 1810 bestehen, vor allem aus zwei Umständen schwer zu beantworten: Zum einen verfügen weder Hugo Chávez noch seine „,bolivarische Bewegung“ über ein klares weltanschauliches Konzept, das ausreichend Kontur besäße, um es einem genaueren Vergleich zu unterziehen. Teilweise wird sogar konstatiert, Chávez verzichte „als Populist“ weitgehend auf eine programmatische Begründung überhaupt ${ }^{12}$. Chávez selber sieht sich zweifellos als Erben des revolutionären Vermächtnisses der venezuelanischen Linken, zu der Chávez vor seiner Wahl zum Staatspräsidenten langjährige, als Offizier auch konspirative Verbindungen unterhalten hatte ${ }^{13}$. Ferner lassen, abgesehen von der persönlichen Beziehung Hugo Chávez' zum kubanischen Staatschef Fidel Castro und der Zusammenarbeit beider Staaten seit 1999 (u.a. im Gesundheitswesen, in der Energieversorgung), bestimmte Herrschaftsmethoden - wie die Errichtung revolutionärer Nachbarschaftsgruppierungen (Círculos Bolivarianos) - auf den Einfluss des kubanischen Revolutionsmodells schließen ${ }^{14}$. Wie Chávez selber ziehen auch einzelne Autoren historische Parallelen zwischen den Befreiungsbewegungen in Venezuela und Kuba im 19. Jahrhundert. Dem „Befreier“ Simón Bolí-

Manuel Caballero, La gestación de Hugo Chávez. 40 años de luces y sombras en la democracia venezolana, Madrid 2000, S.144 f, 149; i.d.S. auch Welsch/Werz, Der Wahlsieg und der Regierungsbeginn von Hugo Chávez Frías (Anm. 4), S. 28 f.

Dies ist die Hauptthese bei Gott, In the Shadow of the Liberator (Anm. 2), S. 17, 19, 27, 45; vgl. ferner den 3. Teil seiner Monographie (S. 125-149), der mit dem Titel „Preparing the overthrow of the ancient régime“ überschrieben ist und Chávez’ Aktivitäten in den Jahren 1992-1998 behandelt.

Welsch/Werz, Der Wahlsieg und der Regierungsbeginn von Hugo Chávez Frías (Anm. 4), S. 28.

Gott, In the Shadow of the Liberator (Anm. 2), S. 31.

14

Monleón, in: Verfassung und Recht in Übersee 37 (2004) (Anm. 4), S. 79. 
var als Vorbild für Hugo Chávez und die „bolivarische Bewegung“ wird der kubanische Patriot José Martí (1853-1895) an die Seite gestellt, auf dessen Kampf gegen die spanische Kolonialherrschaft sich die kubanischen Revolutionäre im 20. Jahrhundert stets berufen haben. ${ }^{15}$

Andererseits lässt gerade die Vielzahl der historischen Vorbilder, die Chávez bemüht, auf eine gewisse Beliebigkeit seiner Konzeption schließen. $\mathrm{Zu}$ den am meisten erwähnten Vordenkern und Vorkämpfern gehören außer Bolívar selber auch dessen Lehrer und Aufklärer, Simón Rodríguez (1769-1852), sowie der Führer der föderalen Streitkräfte, Ezequiel Zamora (1817-1860), der 1859 in der Schlacht von Santa Inés die Landoligarchie der venezuelanischen Llanos besiegte. Chávez beruft sich gern in Reden und pathetischen Ausrufen auf beide Personen ${ }^{16}$. Die politische und weltanschauliche Einstufung der Bewegung Zamoras ist umstritten. Dem Ansatz Hugo Chávez' kommt jene Interpretation entgegen, die die revolutionären Tendenzen der „Guerra Federal“ betont ${ }^{17}$. In diesem Sinne hebt er die Inspiration Zamoras für die heutigen Auseinandersetzungen hervor und legt Wert auf ein durch Zamora bestärktes „,revolutionäres Bewusstsein“. ${ }^{18}$

Eine wieder andere Orientierung gibt der „bolivarischen Bewegung“ der Einfluss des in Lateinamerika umstrittenen argentinischen Politologen und Historikers Norberto Ceresole $^{19}$. Er vertritt eine traditionelle Richtung des Peronismus, fungierte mehrere Jahre als Berater Hugo Chavez' und hat ihn in seinem Interesse für angeblich „fortschrittliche“ lateinamerikanische Militärregierungen beeinflusst ${ }^{20}$. Ein theoretischer Anknüpfungspunkt ist die historische Wechselwirkung von Gesellschaft (sociedad) und Raum (espacio), die hemmend oder fördernd auf das Gelingen einer (länger andauernden) Revolution einwirkten, je nachdem wie die jeweilige Gesellschaft diesen Raum im Sinne ihres Fortschritts

Z.B. Hugo Chávez, Discurso acto de constitución del Frente Nacional en Defensa de la Educación, unter: www.venezuela-oas.org/Speech given at the ceremony to establish the National Front to defend the right to education.pdf, S.1: „Long live Ezequiel Zamora! Long live Simón Rodríguez, teacher!".

So Frederico Brito Figueroa, Tiempo de Ezequiel Zamora, 5. Aufl. Caracas 1981, S. 494 f.

Chávez, Discurso acto de constitución del Frente Nacional (Anm.16), S. 2: „We ratified and extended our revolutionary Zamoran consciousness, because Ezequiel Zamora is the inspiration for todays struggles".

Kontrovers u.a.: Norberto Ceresole, España y los judíos: expulsión, inquisición, holocausto (1492-1997), Madrid 1997, zu den von ihm so genannten , zwei Mythen”, Holocaust und Vertreibung der spanischen Juden, u.a. S. 10 f, $61 \mathrm{ff}$ (,el Mito del Holocausto y la conciencia occidental"). 
nutze $^{21}$. Ceresole gelangt in seiner politischen Analyse zu dem Ergebnis, dass eine (lateinamerikanische) Nation in dem Maße verwundbar und instabil sei, in dem sie sich nicht vor den Gefahren einer zerfallenden Weltgemeinschaft und den damit verbundenen wirtschaftlichen, kulturellen und militärischen Auseinandersetzungen zu schützen wisse. Dies gelte vor allem dann, wenn der Wirtschaftsraum nach außen offen sei und im Inneren keine aufgeklärte Elite besitze, die zu einer entsprechenden Wirtschaftspolitik in der Lage sei ${ }^{22}$. Als Gegenmittel bedürfe es eines neuen ,nationalen Projekts“, das eine Stärkung des nationalen Raums (fortalecimiento del espacio) sichere. In diesem Sinne seien die Elemente wirtschaftliches Handeln (la acción económica), Verteidigung des Vaterlandes (la Defensa de la Patria), auswärtige Politik (la acción exterior del Estado) und die innere staatliche Ordnung zu einer neuen strategischen Ausrichtung der Politik zu verbinden. Als unabdingbare Voraussetzungen hierfür werden die militärische Stärke sowie die konsequente Abkehr von „,neoliberalen“ Wirtschaftskonzepten und den damit verbundenen Privatisierungen angesehen $^{23}$. Hugo Chávez rekurriert in seinen Stellungnahmen wiederholt auf diese Begriffe und den in Lateinamerika insgesamt populären Ansatz. Er verbindet ihn mit Gründermythen, soldatischer und sozial-revolutionärer Romantik sowie autoritären Herrschaftskonzepten zu einem eigentümlichen Weltbild ${ }^{24}$. Kennzeichnend sind Schlagworte wie jene der ,postdemokratischen Zeit“ (posdemocracia) oder der „Wiedergewinnung nationaler Unabhängigkeit“ für Venezuela bzw. Feindbegriffe wie die eines ,,neoliberalen Fundamentalismus“ und einer „Denationalisierung“ Venezuelas, die er bevorzugt auf die „Oligarchie“ des Landes bezieht ${ }^{25}$. Auf der Grundlage des autoritären Konzepts „Führer - Heer Volk“ (caudillo - ejército - pueblo) werden partikulare Einrichtungen und vermittelnde Instanzen abgelehnt, die als politische Parteien, Gewerkschaften, Berufsverbände oder als nach klassischem Muster getrennte unabhängige Staatsfunktionen den Machtanspruch der

Norberto Ceresole, Argentina. Sobre transiciones y decadencias: cinco ensayos geopolíticos para la re-interpretación de la realidad argentina, Madrid 1987, S. 49 ff, insbesondere S. 51.

Am Beispiel Argentiniens: Norberto Ceresole, Subversión, contrasubversión y disolución del poder: guerra y sociedad en la Argentina contemporánea. Un país entrópico en un mundo apolar, Buenos Aires 1996, S. 8.

Ceresole, Subversión, contrasubversión y disolución del poder (Anm. 22), S. 9 ff, 77 ff.

Unter Berufung auf Simón Bolívar u.a. zum militärischen Geist Venezuelas (,,alma militar venezuelana”): Rueda de Prensa del Presidente Hugo Chávez Frías con los Medios Nacionales e Internacionales, Caracas am 15.4.2002, unter: www.venezuela-oas.org/rueda_de_prensa_del_presidente_h.htm.

Hugo Chávez, Discurso en manifestación popular, Caracas am 23.1.2003, unter: www.venezuelaoas.org/Speech given to supporter during a rally in av. Bolívar caracas.pdf, S. 13, zur Gefährdung des revolutionären Prozesses durch ,the predatory, anti-national, privatizing, neoliberal, fascist and coup-plotting oligarchy”; ferner ders., ebda., S. 17, zum Zusammenhang von „Imperialismus”, „Faschismus” und „Neoliberalismus”. 
Staatsführung in Frage stellen können ${ }^{26}$. Schließlich sucht Chávez an die in Lateinamerika alte legalistische Tendenz anzuknüpfen, insofern er den durch ihn selbst vorgelegten Verfassungstext von 1999 glorifiziert: Für immer seien sie alle dieser ,beinahe heiligen Verfassung“" verpflichtet, die er dem Rang nach kaum geringer als die Bibel einstuft. ${ }^{27}$

Zum anderen ist aber auch das Staatsdenken Simón Bolívars nur schwer auf einen für den Vergleich tauglichen Nenner zu bringen. Zu vielschichtig erscheint seine Persönlichkeit, zu schwierig waren die politischen Herausforderungen und viel zu wechselhaft die Ereignisse, als dass sich eine verfassungspolitische Formel bilden ließe oder von Teilaspekten auf ein Gesamtkonzept des „Befreiers“ zu schließen wäre ${ }^{28}$. Ferner spiegeln sich in seinem Denken sehr unterschiedliche weltanschauliche Einflüsse wider, die Bolívar prägten: Das gilt bereits für seine Jugend in Caracas, dessen kreolische Elite zum Ende der Kolonialzeit besonders empfänglich für die europäische, insbesondere französische Aufklärung war, zumeist vermittelt über die konservativere spanische Richtung der ,christlichen Aufklärung“ (cristianismo ilustrado), wie sie Benito Gerónimo Feijóo y Montenegro (1676-1764) repräsentierte. Bolívar war mit dessen Philosophie vertraut, die die scholastische Tradition mit säkularen Elementen verband ${ }^{29}$. Anlässlich mehrerer längerer Aufenthalte zwischen 1799 und 1806 in Europa studierte Bolívar den Typus und die Machtentfaltung des napoleonischen Konsulats und ließ sich in Italien von römisch-republikanischen Idealen inspirieren $^{30}$. Der enttäuschende Eindruck, den die vernachlässigten Städte und Dörfer in Spanien, die wirtschaftliche Lage der Halbinsel, der Niedergang des spanischen Absolutismus sowie die Dekadenz und Korruption am Madrider Hof auf Bolívar machten, trug erheblich zur Korrektur seines Spanienbildes von der „mächtigen Metropole“ bei. Umgekehrt beeindruckten ihn in Frankreich Wohlstand und Ordnung, die er als „Früchte der Freiheit“ einzig

Caballero, La gestación de Hugo Chávez (Anm. 10), S. 151 f; Welsch/Werz, Der Wahlsieg und der Regierungsbeginn von Hugo Chávez Frías (Anm. 4), S. 32.

„Siempre, siempre, y para siempre apegados a esta casi sagrada Constitución, después de la Biblia la Constitución Bolivariana de Venezuela", Hugo Chávez, Cadena Nacional Restitución de Poderes, Caracas am 14.4.2002, unter: www.venezuela-oas.org/cadena_nacional_restitución_de_p. htm.

Tomás A. Polanco, Prólogo, in: Pedro Grases (Hg.), El Libertador y la Constitución de Angostura de 1819, Caracas 1970, S. 10.

29

Den Einfluß Feijóos betont besonders Salvador de Madariaga, Bolívar, London 1952, S. 78 f; dazu ferner Stoetzer, in: Stegmann, Simón Bolívar (Anm. 3), S. 59 f; Ulrich K. Preuss, Der Feldherr als Verfassungsgeber, in: Simón Bolívar, Rede von Angostura am 15. Februar 1819, Hamburg 1995, S. 62 f.

30 Stoetzer, in: Stegmann, Simón Bolívar (Anm. 3), S. 60 ff; Preuss, in: Bolívar, Rede von Angostura am 15. Februar 1819 (Anm. 29), S. 64 f. 
in der Staatsform der Republik verwirklicht $\operatorname{sah}^{31}$. Er zog die Schlussfolgerung, dass es eines entsprechenden institutionellen Rahmens auch deshalb bedürfe, damit ein Einzelner seine Genialität in dem Maße entfalten könne, wie es dem Konsul Napoleon gelungen war. Gerade Napoleons Beispiel hat Bolívar in seinen militärischen und politischen Überzeugungen geprägt, was in seinen Heeresbekanntmachungen zum Ausdruck kommt ${ }^{32}$. Der Parallelen zu Napoleon wegen ist in der biographischen Literatur davon gesprochen worden, Bolívar sei nur ,als Originalkopie der napoleonischen Urform“ wirklich zu begreifen. $^{33}$

Andererseits wird darauf hingewiesen, dass Bolívar in seinem Idealismus Anstoß an der zunehmenden Machtfülle und dem Herrschaftsverständnis Napoleons nahm, die er spätestens nach dessen Erhebung zum Kaiser als sichere Voraussetzung für eine künftige neue Tyrannis ansah $^{34}$. Jedenfalls verrät Bolívars Auffassung von Staatsorganisation das französische Vorbild, am stärksten in den bolivarischen Verfassungen der Staaten Bolivien und Peru von 1826: Die klassische Dreiteilung der Funktionen (oder auch Gewalten) wird zur Vierteilung mit einer elektoralen, legislativen, exekutiven und judikativen Gewalt erweitert. Die „Wählergewalt“ (poder electoral) geht auf die Verfassungen Napoleons der Jahre VIII und X zurück und auf die Konzeption einer ,,neutralen Gewalt“ (pouvoir neutre) bei Benjamin Constant, die erst Napoleon und nach ihm Bolívar 1826 in jene Wählergewalt transformierten $^{35}$. Ebenfalls auf das napoleonische Vorbild geht die Einrichtung einer weiteren Kammer zurück, der Zensorenkammer. Die Zensoren in Bolívars Konzept wurden wie die Senatoren Napoleons auf Lebenszeit ernannt; sowohl die bolivarische Kammer der Zensoren wie auch der französische Senat hatten Funktionen im Sinne einer ,republikanischen

Gilette Saurat, Bolívar le Libertador, Paris 1979, S. 38, 47; ferner Donald E. Worcester, Bolívar, London 1978, S. 13.

U.a. Ernennung zum Generalkapitän der Armee mit dem Titel „Libertador“ vom 14. Oktober 1813; Wahl und Übernahme der höchsten diktatorischen Macht vom 2. Januar 1814; Errichtung der „Guardia del Libertador“ vom 18. Mai 1822; Stoetzer, in: Stegmann, Simón Bolívar (Anm. 3), S. $66 \mathrm{f}$.

Madariaga, Bolívar (Anm. 29), S. XV.

34 Übereinstimmend Emil Ludwig, Bolívar. The Life of an idealist, London 1947, S. 36 f; Francisco Cuevas Cancino, Bolívar en el tiempo, Mexiko, 2. Aufl. 1982, S. 94; Ricardo Herren, Bolívar, Barcelona 1994, S. 43 f; abweichend Madariaga, Bolívar (Anm. 29), S. 65 ff: Die wiederholte Kritik Bolívars habe nichts an seiner Bewunderung für Napoleon und an dessen Vorbildwirkung geändert; i.d.S. auch Gerhard Masur, Simón Bolívar und die Befreiung Südamerikas, Konstanz 1949, S. $60 \mathrm{ff}$.

Simón Bolívar, Discurso del Libertador al Congreso Constituyente de Bolivia (Lima, 25. Mai 1826), in: ders., Discursos, proclamas y epistolario político, Madrid 1975, S. 300: "El proyecto de constitución ... está dividido en cuatro poderes políticos, habiendo añadido uno más, sin complicar por esto la división clásica de cada uno de los otros“; zum Einfluss der napoleonischen Konsulatverfassung: Masur, Simón Bolívar (Anm. 34), S. 525. 
Inquisition“, die die Gesetze und die öffentliche Moral überwachen sollte ${ }^{36}$. Diese Konzeption hat Hugo Chávez zu einer seiner markantesten Neuerungen in der Verfassung von 1999 inspiriert, worauf noch näher einzugehen ist.

In weltanschaulicher und verfassungsrechtlicher Hinsicht hat Bolívar in jeder Phase seines Wirkens auf Konzepte der europäischen Aufklärung rekurriert: Mit Bolívars Idealismus und dem Einfluss seines Lehrers, Simón Rodríguez, lässt sich begründen, dass Jean-Jaques Rousseau und die politische Romantik besonderen Einfluss auf Bolívar ausübten ${ }^{37}$. Bolívar besaß den „Gesellschaftsvertrag“ und betonte die Nützlichkeit der Schrift und seine Wertschätzung für deren Autor, allerdings mit der Einschränkung, dass dessen Werk nicht nur Gutes, sondern auch Schlechtes bewirkt habe ${ }^{38}$. Für die weltanschauliche Differenz Bolívars zu Rousseau spricht auch, dass letzterer ein abstraktes Freiheitsprinzip vertrat und angesichts der angeblichen Verderbnis der Gesellschaft alle Hoffnung auf die Bestimmung der Individuen zum Guten setzte, was aber Bolívars praktischen Erfahrungen widersprach: Er misstraute der politischen Einsichtsfähigkeit der Individuen und war daher bemüht, gesellschaftliche Kräfte zu ermutigen und institutionelle Gegengewichte zu installieren, die einer befürchteten Anarchie entgegenwirken sollten ${ }^{39}$. Was das Verfassungskonzept Bolívars betrifft, überwiegen daher klar die Bezüge zu Charles de Montesquieu: Dies gilt nicht nur wegen der Bedeutung, die dessen Lehre von der Gewaltenteilung und die Abkehr vom Prinzip der monarchischen Souveränität für den frühen Konstitutionalismus überhaupt besa ${ }^{40}$. Bolívar machte sich den Standpunkt Montesquieus auch in einer Erkenntnis zu eigen, die den Emanzipationsbestrebungen in Übersee sehr entgegen kam: Soweit ein Land über jeweils spezifische (physische, geographische, kulturelle) Lebensbedingungen verfüge, müsse es seine eigene staatliche Form finden. Die Lebensbedingungen korrespondierten mit gesellschaftlichen Gesetzmäßigkeiten, die von Volk zu Volk verschieden und nicht verallgemeinerungsfähig seien. Insofern habe sich auch die Gesetzgebung an den natürlichen Gegebenheiten, an der „Natur der Sache“ zu orientieren ${ }^{41}$. In diesen Bedingun-

Näher Stoetzer, in: Stegmann, Simón Bolívar (Anm. 3), S. 67, 69.

Ludwig, Bolívar (Anm. 34), S. 15 ff; Masur, Simón Bolívar (Anm. 34), S. 44.

Simón Bolívar, Brief vom 15.11.1824 an General Robert Wilson, in: R. Blanco-Fombona (Hg.), Cartas de Bolívar 1823-1824-1825, Madrid 1921, S. 249.

Masur, Simón Bolívar (Anm. 34), S. 12, 346; Madariaga, Bolívar (Anm. 29), S. 79.

Charles de Montesquieu, De l'Esprit des lois (1748), 11. Buch, 6. Kap., Bd. 1, Paris 1979, S. 294 ff. So stellte Bolívar in seiner Rede vom 15. Februar 1819 auf dem zweiten Nationalkongress Venezuelas in Angostura die Lehre Montesquieus, v.a. in Bezug auf die Gewaltenteilung, in den Mittelpunkt, Bolivar, Discurso pronunciado por el Libertador ante el Congreso de Angostura el 15 de febrero de 1819, día de su instalación, in: ders., Discursos, proclamas (Anm. 35), S. 230 ff.

Montesquieu, De 1'Esprit des lois (Anm. 40), 1. Buch, 1. und 2. Kap., Bd. 1, S. 123 ff; 12. Buch, 4. Kap., Bd. 1, S. 329. 
gen unterscheide sich Spanischamerika aber in klimatischer, ethnischer und kultureller Hinsicht in einem solchen Maße von Europa - wie auch von Nordamerika - , dass eine Übernahme fremder Muster und eine Staats- und Rechtseinheit mit Spanien von vornherein ausgeschlossen sein mussten und Bolívar für die solcherart spezifische venezuelanische Gesellschaft eine eigene staatliche Form fordern konnte ${ }^{42}$. Bereits in diesen beiden Punkten kommt ein Merkmal des bolivarischen Staatsdenkens zum Ausdruck, das durchaus stellvertretend für einen in ganz Lateinamerika bis heute weit verbreiteten Eklektizismus steht: Nicht ganze Lehrgebäude werden übernommen, sondern recht beliebig einzelne Teilaspekte aus sehr unterschiedlichen Quellen, die die politischen Forderungen zu untermauern geeignet sind.

Noch deutlicher wird dies in der Adaption Benjamin Constants, der zur Zeit der Europareisen Bolívars die staatsrechtlichen Debatten in Frankreich maßgeblich prägte: Seine Lehre von der ,gemäßigten Gewalt“ (pouvoir moderé), die die Konsequenzen aus den Fehlentwicklungen der Französischen Revolution zog, machte sich Bolívar in einem sehr maßgeblichen Punkte zu eigen: Er schlug auf dem Kongress von Angostura 1819 neben den drei bekannten Staatsfunktionen (oder Gewalten) Exekutive, Legislative und Judikative eine neue vierte Funktion vor, die ,moralische Gewalt“ (poder moral). Sie sollte aus zwei Kammern bestehen („Cámara de Moral“ und „Cámara de Educación“) und rekurrierte ganz im klassischen und moralisierenden Zeitgeist auf die altgriechische Einrichtung des Aeropags und die römischen Zensoren ${ }^{43}$. Ebenfalls der Lehre Constants, aber auch dem nordamerikanischen Muster verpflichtet ist die Überzeugung Bolívars, dem Senat als unabhängige Einrichtung eine ausgleichende, harmonisierende Funktion zwischen den übrigen Gewalten zu verleihen. Diese die Leidenschaften einerseits und das Übergewicht einzelner Staatsfunktionen andererseits mäßigenden, hemmenden Vorkehrungen sind nicht zu trennen vom Tugendverständnis Bolívars. Es findet in dieser vierten Gewalt als Wächterin der öffentlichen Moral eine institutionelle Entsprechung - auch wenn der Kongress von Angostura 1819 schließlich nicht seinem Vorschlag zur Institutionalisierung der vierten Gewalt folgte. Da Bolívar die republikanische Gemeinschaft tugendhafter Bürger infolge einer Zerrissenheit der Gesellschaft für gefährdet hielt, musste diese Tugend mit staatlicher Autorität durchgesetzt werden, wiewohl diese Autorität ihrerseits institutionell zu bändigen sei. Der Umstand, dass Bolívar eine weitere Stärkung der Exekutive forderte und - wie dann in den Verfassungen von 1826 verankert - sogar eine Präsidentschaft auf Lebenszeit

Bolívar, Discurso pronunciado por el Libertador ante el Congreso de Angostura, in: ders., Discursos, proclamas (Anm. 35), S. 223 f., mit Bezug auf Montesquieus „Geist der Gesetze“.

43

Bolivar, Discurso pronunciado por el Libertador ante el Congreso de Angostura, in: ders., Discursos, proclamas (Anm. 35), S. 240: „Tomemos de Atenas su areópago y los guardianes de las costumbres y de las leyes; tomemos de Roma sus censores y sus tribunales domésticos"; zu den Motiven ferner Masur, Simón Bolívar (Anm. 34), S. 344 f. 
(presidencia vitalicia) befürwortete, lässt sich eben damit erklären, dass er im Verlaufe des Emanzipationsprozesses immer skeptischer gegenüber der Selbstordnungsfähigkeit der Gesellschaft geworden war ${ }^{44}$. Schließlich ließ er sich wie in Peru (1826) auch in Kolumbien (1828) zum Diktator ausrufen.

Anders als in der Bewertung dieser autoritären Tendenzen, besteht in der Literatur weitgehend Einigkeit darüber, dass der Pragmatismus überwog und Bolívar darauf abzielte, in politischen Fragen praktikable, lebensnahe Lösungen zu finden ${ }^{45}$. In verfassungsrechtlicher Hinsicht wollte er seine politische Organisation nicht an einem gesellschaftlichen Ideal ausrichten, sondern daran, wie die Gesellschaft tatsächlich beschaffen war ${ }^{46}$. Rezeptionswege im Einzelnen zu erhellen, wird noch zusätzlich dadurch erschwert, dass sich alle diese ohnehin vielfältigen Theorien, Rechtsfiguren und Verfassungsmuster bei Simón Bolívar mit seiner Bewunderung für die Traditionen der englischen Rechts- und Institutionengeschichte verbanden. Diese Anziehungskraft war durchaus zeitgemäß, wenn man den Einfluss des englischen Verfassungsdenkens auf zahlreiche spanischamerikanische Liberale der Emanzipationsphase, im besonderen aber auf die venezuelanische Elite dieser Zeit berücksichtigt $^{47}$. Eine nicht unerhebliche Rolle spielten hierbei die politischen Erwartungen an Großbritannien, das einerseits mit Spanien im Kampf gegen Frankreich $(1808$ - 1813) verbündet war, von dem sich andererseits aber die Spanischamerikaner - so auch die jungen venezuelanischen Kreolen um Simón Bolívar, Luis López Méndez und Andrés Bello - eine Unterstützung der Emanzipationbestrebungen gegenüber der spanischen Krone erwarteten ${ }^{48}$. England galt Bolívar, wozu seine diplomatischen Missionen nach London und seine Lektüre beitrugen, als Muster für neuzeitliche Verfassungen schlechthin, ebenso wie er die römische Staatsorganisation als repräsentativ für das klassische Altertum hielt. Seine Neigung, sich wahlweise sehr verschiedener Vorbilder zu bedienen, kommt darin zum Aus-

Bolívar, Discurso del Libertador al Congreso Constituyente de Bolivia, in: ders., Discursos, proclamas (Anm. 35), S. 303, 306; ferner Preuss, in: Bolívar, Rede von Angostura am 15. Februar 1819 (Anm. 29), S. 98. Den Vorschlag, eine Person auf Lebenszeit als ,elective monarch“ mit der exekutiven Gewalt zu betrauen, hatte (erfolglos) bereits Alexander Hamilton im Juni 1787 dem verfassunggebenden Konvent von Philadelphia unterbreitet; dazu Harald v. Bose, Republik und Mischverfassung - zur Staatsformenlehre der Federalist Papers, Frankfurt/M. 1989, S. 79. Madariaga, Bolívar (Anm. 29), S. 77: , After all, Bolívar was not a thinker, but a man of action. His natural trend was not to build up a coherent image of the world as a system". Polanco, in: Grases, El Libertador y la Constitución de Angostura de 1819 (Anm. 28), S. 13. Speziell für die Venezuelaner: Iván Jaksić, Andrés Bello: Scholarship and Nation-Building in Nineteenth-Century Latin America, Cambridge 2001, S. $30 \mathrm{ff}$.

Das Vorbringen der venezuelanischen Gesandtschaft ist dokumentiert: Los Comisionados de la Junta de Gobierno de Caracas ante el Gabinete Británico, Simón Bolívar y Luis López Méndez, dan cuenta al secretario de Relaciones Exteriores de Venezuela del desarrollo de su misión y de los esfuerzos hechos para ,producir la emancipación general“ del continente. Londres, 8 de septiembre de 1810, in: Simón Bolívar, Doctrina del Libertador, 2. Aufl., Caracas 1976, S. 4 ff. 
druck, dass Bolívar Großbritannien trotz seiner monarchischen Staatsform jedenfalls in der Substanz für eine Republik hielt, insofern diese Verfassungsordnung Volkssouveränität, Gewaltenteilung und Freiheitsrechte miteinander verband und als solche unter der ,rule of law“ stand ${ }^{49}$. Darauf weist umgekehrt aber auch der Umstand hin, dass Bolívar das nordamerikanische Modell gering schätzte, obwohl es tatsächlich erheblichen Einfluss auf alle venezuelanischen Verfassungen seit 1811 hatte, auch auf die beiden bolivarischen von 1819 und 1821. Auch hier dachte Bolívar viel eher praktisch und viel weniger theoretisch, wenn er das Modell der englischen konstitutionellen Monarchie in einen republikanischen Rahmen übertragen wollte: ein Zweikammersystem in Abkehr vom Einkammersystem der spanischen Verfassung von Cádiz 1812; eine parlamentarische Vertretung der gesellschaftlichen Elite in einer eigenen Kammer - dem Senat -, wenn auch nicht des Adels wie im englischen Oberhaus; die Verehrung und das Prestige eines Staatsoberhauptes, das diesen zum ruhenden Pol angesichts der Parteienkämpfe und äußeren Bedrohungen machen könne, ohne dass damit die monarchische Erbfolge einer Dynastie verbunden wäre. ${ }^{50}$

\section{Die Verfassungsentwürfe Simón Bolívars}

Kennzeichnend für den frühen Konstitutionalismus in Spanischamerika im allgemeinen und in Venezuela im besonderen ist eine rasche Abfolge sehr verschiedener, in mancher Hinsicht widersprüchlicher Verfassungsentwürfe und -texte, die die politische Instabilität und weltanschauliche Desorientierung zu Beginn der Emanzipationsbestrebungen widerspiegeln. Den ersten Schritt machte der Stadtrat (cabildo, ayuntamiento) von Caracas, der sich im Frühjahr des Jahres 1810 nach dem Vorbild der revolutionären spanischen Ausschüsse (juntas) für die Provinzen des Generalkapitanats Venezuela als „oberster, die Rechte Ferdinands VII. schützender Ausschuss“ (Suprema Junta Conservadora de los Derechos de Fernando VII) proklamierte. Dieser Ausschuss anerkannte zumindest formal die Legitimität des im französischen Exil weilenden Königs, lehnte aber die Autorität der provisorischen spanischen Regierung (Regencia) ab und beanspruchte für die Einwohner des Generalkapitanats die Ausübung der Souveränität, die nach den Grundsätzen der alten spanischen Verfassung an das Volk zurückgefallen sei $^{51}$. Damit war der Weg zur Einberufung einer ersten verfassunggebenden Versammlung beschritten. Sie verkündete wie kurz zuvor die

49

50

Preuss, in: Bolívar, Rede von Angostura am 15. Februar 1819 (Anm. 29), S. 95. S. $69 \mathrm{f}$.

Acta de Instalación de la Junta Suprema de Venezuela del 19 de abril de 1810, in: Allan R. Brewer-Carías (Hg.), Las Constituciones de Venezuela, Madrid 1985, S. 157: „Ejerciendo los derechos de la soberanía, que por el mismo hecho ha recaido en el pueblo, conforme a los mismos principios de la sabia constitución primitiva de la España“. 
spanische Konstituante von Cádiz (1810) die volle Souveränität und die Vertretung des Volkes und verband diese Proklamation mit der feierlichen Erklärung der Unabhängigkeit Venezuelas vom 5. Juli $1811^{52}$. Die am 21.12.1811 verabschiedete Verfassung fasste wesentliche Prinzipien ihres nordamerikanischen Vorbilds von 1787 mit solchen der Französischen Revolution zusammen: Die Entscheidung für eine bundesstaatliche Ordnung ging mit sehr weitreichenden Befugnissen einher, die die früheren Provinzen nach dem Muster der nordamerikanischen Bundesstaaten zu weitgehend unabhängigen (Teil)staaten machten. Die in der „Erklärung der Rechte des Volkes“ vom 1. Juli 1811 verabschiedeten Rechtgarantien, u.a. der rechtlichen Gleichheit, des Eigentums, der Wirtschaftsfreiheit, bezogen sich einerseits auf die französische Erklärung der Menschen- und Bürgerrechte, reflektierten andererseits die liberale Weltanschauung der kreolischen Oberschicht und entsprachen deren Bedürfnis nach Absicherung ihrer Sonderstellung und ihrer wirtschaftlichen Position gegenüber den übrigen Bevölkerungsgruppen.

Auch Bolívar vertrat diese Konzeption bis zuletzt in seiner Hervorhebung der bürgerlichen Freiheit (libertad civil), der persönlichen Sicherheit (seguridad personal), des unverletzlichen Eigentums (propiedad) und der Gleichheit, die allen übrigen Garantien vorausgehe. Gleichheit (Igualdad) ist allerdings noch nicht als sozialstaatliches Gleichheitspostulat zu verstehen, sondern als Gleichheit vor dem Gesetz, wie gerade Bolívars Kritik an der Sklaverei verdeutlicht. ${ }^{53}$

In der Literatur ist auf den aristokratischen Grundzug bei Bolívar und der kreolischen Elite Venezuelas hingewiesen worden, auch wenn ihre Entscheidung zugunsten der staatlichen Emanzipation nach politischen Maßstäben revolutionär war und sie sich zur Begründung auf die französischen Aufklärer des 18.Jahrhunderts beriefen ${ }^{54}$. Dieses elitäre Verständnis tritt im mehrstufigen Zensuswahlrecht zutage, das Bestandteil der nachfolgenden bolivarischen Verfassungen von 1819 und 1821 war. Ganz im Sinne des liberalen Zeitgeistes begründete Bolívar sein Konzept, das weiter zwischen aktiven (wahlberechtigten) und passiven Bürgern (ciudadanos activos y pasivos) unterscheidet, mit der überragenden Bedeutung der zu schützenden Güter Arbeit und Wissen sowie mit der politischen Notwendigkeit, den in allgemeinen Wahlen zum Ausdruck kommenden demokratisch-revolutionären Tendenzen entgegenzuwirken ${ }^{55}$. Die Zuspitzung des Unabhängigkeitskrieges zwischen

Declaración solemne de la Independencia por el Congreso de Venezuela, in: Brewer-Carías, Las Constituciones de Venezuela (Anm. 51), S. $171 \mathrm{ff}$.

Bolivar, Discurso del Libertador al Congreso Constituyente de Bolivia, in: ders., Discursos, proclamas (Anm. 35), S. 308.

54

Worcester, Bolívar (Anm. 31), S. 23.

55

Bolivar, Discurso pronunciado por el Libertador ante el Congreso de Angostura, in: ders., Discursos, proclamas (Anm. 35), S. 241. 
1812 und 1819, verbunden mit zahllosen Opfern und schweren Verwüstungen, verhinderte auch in verfassungsrechtlicher Hinsicht eine Kontinuität zwischen dem ersten Anlauf zu einer republikanischen Staatsordnung (1810-1812) und der weiteren Entwicklung politischer Institutionen, die der Kongress von Angostura (1819) einleitete. ${ }^{56}$

Einen französisch-revolutionären Einfluss verrät die Entscheidung der Konstituante im Jahr 1811, die Exekutive auf ein Kollegium mit wechselndem Vorsitz aufzuteilen. Bolívar, der während dieser ersten republikanischen Periode diplomatische und militärische Aufgaben wahrnahm, wies der Verfassung von 1811 einen erheblichen Anteil am baldigen Scheitern der ersten Republik im Jahr 1812 zu. In seinen ersten grundlegenden verfassungsrechtlichen Stellungnahmen, die er unter dem Eindruck militärischer Rückschläge im kolumbianischen Cartagena bzw. dann auf Jamaika verfasste, zog er - hierin wegweisend für die folgenden Verfassungen - eine doppelte Schlussfolgerung: Mangels politischer Reife und gesellschaftlicher Homogenität der Bevölkerung, aber auch angesichts der schwierigen äußeren Umstände seien sowohl der Föderalismus wie auch eine schwache Exekutive völlig ungeeignet für eine zukünftige Staatsordnung ${ }^{57}$. Ein in diesen Reden - aber auch im Staatsdenken Bolívars insgesamt - zu Tage tretender Widerspruch besteht zwischen seiner tiefen Skepsis gegenüber den Fähigkeiten der Menschen zur Selbstregierung auf der einen und seinem großen Idealismus und weit gespannten Plänen für eine staatliche Neuordnung auf der anderen Seite. Dies findet eine Entsprechung in der dramatischen Zuspitzung seiner Verfassungskonzepte und schlägt sich nieder in der ,,immer wieder beschworenen Alternative zwischen Anarchie und Despotismus, vor der nur die Gesetzmäßigkeit der Herrschaft, das heißt eine republikanische Verfassung bewahren könne“ ${ }^{58}$. In Bolívars Konzept der Gewaltenteilung (división de poderes) überwiegt der Aspekt der gegenseitigen Hemmung und ihres Ausgleichs (equilibrio de poderes) gegenüber dem einer klaren Trennung und Zuteilung der Funktionen (oder Gewalten). Dies wird insbesondere in der Aufteilung der Legislative auf mindestens zwei, bevorzugt aber drei gesetzgebende Körperschaften deutlich $^{59}$. Bolívar teilte mit den nordamerikanischen Verfassungsvätern die Sorge vor den Folgen einer direkten Demokratie und eines demokratischen Absolutismus, sobald ein vom Volk gewähltes Organ alle Gewalt auf sich vereinigte. Dies erklärt seine Vorliebe für ein

Polanco, in: Pedro Grases, El Libertador y la Constitución de Angostura de 1819 (Anm. 28), S. $10 \mathrm{f}$.

Bolívar, Memoria dirigida a los ciudadanos de la Nueva Granada por un caraqueño (sog. Manifest von Cartagena vom 15. Dezember 1812), in: ders., Discursos, proclamas (Anm. 35), S. 40 ff; Contestación de un americano meridional a un caballero de esta isla (Kingston, 6. September 1815, sog. Brief aus Jamaika), in: ders., ebda., S.160 ff, $166 \mathrm{ff}$.

Preuss, in: Bolívar, Rede von Angostura am 15. Februar 1819 (Anm. 29), S. 94; den persönlichen Zwiespalt betont ferner Madariaga, Bolívar (Anm. 29), S. 71, 80 ff. 
Zweikammersystem und eine durch Wahlmännerentscheidung und Zensuswahlrecht gekennzeichnete Auswahl der Repräsentanten. Nicht nur die Abgeordneten einer zweiten Kammer, sondern auch die starke Stellung des Präsidenten als Staats- und Regierungschef sollten als hemmende, mäßigende Elemente der „Zügellosigkeit“ und Erregbarkeit des Demos entgegenwirken. Bolívar schwebte ein System vor, das Einheit und Stabilität in einer unitarischen Staatsordnung gewährleistete, dabei aber dem Einzelnen so viel individuelle Freiheit wie möglich sicherte. Die Dichotomie zwischen Effizienz des Staates und Freiheit ist grundlegend für Bolívars Verfassungsverständnis ${ }^{60}$. Die Verfassung von 1819 und kurz darauf jene von 1821 sollten in institutioneller Hinsicht dieses Konzept umsetzen:

- Die durch den Kongreß (Congreso general de Venezuela) wahrgenommene legislative Funktion wird aufgeteilt auf Repräsentantenhaus (Cámara de Representantes) und Senat (Senado); wobei die Gesetzesinitiative ebenso wie Änderungsvorschläge oder Einsprüche grundsätzlich von jeder der beiden Kammern ausgehen können ${ }^{61}$. Das in Bolívars ersten Verfassungsentwürfen noch eindeutige Bekenntnis zur Gewaltenteilung kommt in weiteren Befugnissen des Kongresses zum Ausdruck: Die Möglichkeit der Anklage des Präsidenten durch das Repräsentantenhaus und der übrigen hohen Funktionsträger des Staates vor dem Senat $^{62}$; ferner die Zustimmung des Kongresses zum Abschluss völkerrechtlicher Verträge ${ }^{63}$. Der konservativ-aristokratische Ansatz Bolívars wird in seinem Vorschlag an den Kongress von 1819 deutlich, die Funktion des Senators einerseits die gesamte Lebenszeit ausüben und lediglich die erstmalige Ernennung durch den verfassunggebenden Kongress von Angostura vornehmen zu lassen ${ }^{64}$; sowie andererseits das Senatorenamt erblich zu machen ${ }^{65}$. Der Kongress von Angostura lehnte dieses zweite aristokratische Element ab und beließ es bei einer Ausübung des Senatorenamtes auf Lebenszeit. Außerdem entschied sich der Kongress gegen Bolívars Wunsch, eine weitere, vierte Gewalt (Poder Moral)

60

61

62

63

\author{
6. Titel, 1. Sektion, Art. 7 Abs. 17 f der Verfassung von 1819; Art. 55 Abs. 17 f der Verfassung \\ von 1821; in der Verfassung von 1811 war diese Befugnis dem Senat noch alleine vorbehalten \\ (Art. 93).
}

64

65

Worcester, Bolívar (Anm. 31), S. 4 f; ähnlich Masur, Simón Bolívar (Anm. 34), S. 12, 265 f.

6. Titel, 1. Sektion, Art. 1 f, Art. 7 Abs. 1 der Verfassung von 1819; Art. 40 f der Verfassung von 1821; so auch zuvor Art. 3 f der Verfassung von 1811.

7. Titel, 5. Sektion, Art. $1 \mathrm{ff}$ bzw. 6. Titel, 3. Sektion, Art. 9 ff der Verfassung von 1819; Art. $89 \mathrm{f}$ der Verfassung von 1821; ähnlich bereits Art. 52 der Verfassung von 1811; Vorbild war das Impeachment-Verfahren der nordamerikanischen Verfassung von 1787.

6. Titel, 3. Sektion, Art. 2 f der Verfassung von 1819

So 6. Titel, 3. Sektion, Art. 2 Proyecto de Constitución para la República de Venezuela, formado por el Jefe Supremo, y presentado al Segundo Congreso Constituyente para su examen, in: Grases, El Libertador y la Constitución de Angostura de 1819 (Anm. 28), S. 158: „Las funciones de Senador serán vitalicias y hereditarias". 
einzurichten, die als pädagogische Instanz und Sittengericht, letztlich als Ergänzung und Gegengewicht zu den herkömmlichen drei Staatsfunktionen dienen sollte. ${ }^{66}$

- Um, wie von Bolívar angemahnt, die Stellung der Exekutive zu befestigen, wandte sich die erste bolivarische Verfassung von 1819 gegen die Vorläuferkonzeption, wonach ein vom Volk gewählter Exekutivrat für vier Jahre die ausübenden Befugnisse wahrgenommen hatte $^{67}$. Diese Befugnis wird nunmehr durch den Präsidenten ausgeübt, womit sich das für die weitere südamerikanische Verfassungsgeschichte charakteristische Präsidialsystem durchsetzte. Der Präsident beruft den Kongress zur neuen, jährlichen Sitzungsperiode ein $^{68}$. Er ernennt außer den Mitgliedern des Kabinetts auch die übrigen hohen Beamten ${ }^{69}$ und fungiert nicht nur als Chef der Staatsverwaltung, sondern gewährleistet in Person die Aufrechterhaltung der Ordnung, inneren und äußeren Frieden ${ }^{70}$. Zu diesem Zweck darf der Präsident den Ausnahmezustand verhängen, wenn auch der Kongress - gegebenenfalls erst nachträglich - mit dieser Entscheidung zu befassen ist ${ }^{71}$. Der Präsident hat schließlich Anteil an der Gesetzgebung, da ein Gesetz erst mit Gegenzeichnung der Exekutive zustande kommt und im Falle eines Vetos einer erneuten Abstimmung und der Annahme mit Zweidrittelmehrheit bedarf ${ }^{72}$. Bolívars Empfehlung, den Präsidenten für eine längere Amtszeit von sechs Jahren zu wählen ${ }^{73}$, ging der Konstituante 1819 zu weit. Sie beließ es bei einer vierjährigen Amtszeit.

- Bolívar bevorzugte ein gestuftes Wahlrecht und eine Wahl der Abgeordneten über Wahlmänner ${ }^{74}$. Beide Vorkehrungen gegen unmittelbare, allgemeine und gleiche Abstimmungen des Volkes haben Eingang in die Verfassungen von 1819 und 1821 gefunden. Die

So die Anlage zum Verfassungsentwurf: Apéndice a la Constitución relativo al Poder Moral, in: Grases, El Libertador y la Constitución de Angostura de 1819 (Anm. 28), S. 197 ff.

67

I.E. Art. $72 \mathrm{ff}$ der Verfassung von 1811.

68

6. Titel, 3. Sektion, Art. 3 der Verfassung von 1819; Art. 115 der Verfassung von 1821.

69

7. Titel, 3. Sektion, Art. 11 der Verfassung von 1819 ohne, dass von einer Zustimmung des Kongresses die Rede ist; dagegen schreibt Art. 121 der Verfassung von 1821 die Zustimmung des Kongresses vor.

7. Titel, 3. Sektion, Art. 4 f der Verfassung von 1819; Art. 113 der Verfassung von 1821.

71 7. Titel, 3. Sektion, Art. 20 der Verfassung von 1819; ähnlich Art. 128 der Verfassung von 1821, allerdings mit einer klareren Abgrenzung der Entscheidungsbefugnisse und rascheren Befassung des Kongresses.

6. Titel, 1. Sektion, Art. 11 f der Verfassung von 1819; Art. 46 f der Verfassung von 1821; ferner bereits Art. 9 der Verfassung von 1811.

73 7. Titel, 1. Sektion, Art. 3 des Verfassungsentwurfs; eine Präsidentschaft auf Lebenszeit verfolgte Bolívar in Angostura noch nicht, Masur, Simón Bolívar (Anm. 34), S. 344.

74

4. Titel, 1. u. 2. Sektion des Verfassungsentwurfs (1819). 
aktiven Wahlbürger der Gemeinden wählen aus ihrem Kreis in dafür vorgesehenen Versammlungen (asambleas parroquiales) neben den Gemeinderäten und Friedensrichtern die Wahlmänner der Gemeinden (electores parroquiales) ${ }^{75}$. Diese versammeln sich in den Hauptstädten ihrer jeweiligen Departements und benennen je nach Bevölkerungszahl des Departements einen oder mehrere Repräsentanten für den Kongress ${ }^{76}$. Die Eigenschaft des Aktivbürgers, des Wahlmannes und des Abgeordneten sind an bestimmte qualifizierende Anforderungen gebunden, insbesondere Einkünfte in einer bestimmten Höhe, (Grund)eigentum oder einen herausgehobenen beruflichen Status. ${ }^{77}$

- Bolívar teilte die Position nicht nur der kreolischen Elite seiner Heimat, sondern auch der liberalen Staatsmänner dieser Zeit in Europa und Nordamerika zum absoluten Schutz des Privateigentums. In diesem liberalen Sinne entspricht die Verfassung des Jahres 1819 wortgleich der Eigentumsverbürgung von 1811: „Eigentum meint das Recht, nach Belieben seine Güter und die Früchte seiner Talente, seines Fleißes und seiner Arbeit zu nutzen und darüber zu verfügen “78. Diesen Wortlaut hatte auch Bolívar für seinen Verfassungsentwurf gewählt ${ }^{79}$. Hervorzuheben ist insbesondere der ausdrückliche Bezug auf das für die Rechtsordnungen der Neuzeit maßgebliche Konzept des englischen Philosophen John Locke: Das Eigentum ist in dem Maße höchstpersönlich und unbedingt zu schützen, in dem es auf dem Einsatz der individuellen Tatkraft und Kreativität beruht ${ }^{80}$. Nur im Ausnahmefall einer besonderen, gesetzlich festgelegten „öffentlichen Notwendigkeit“ (necesidad pública) oder allgemeinen Nützlichkeit (,utilidad general“) und gegen eine angemessene Entschädigung ist eine Enteignung zulässig ${ }^{81}$. In die gleiche Richtung zielte auch der absolute Schutz der

4. Titel, 1. Sektion, Art. 2 ff der Verfassung von 1819; ebenso mit etwas abweichenden Bezeichnungen Art. 12 ff, Art. 22 der Verfassung von 1821; zuvor bereits Art. 14 ff der Verfassung von 1811.

4. Titel, 2. Sektion, Art. 1 ff der Verfassung von 1819; Art. 30 f der Verfassung von 1821; ferner Art. 22 f, Art. 76 der Verfassung von 1811.

4. Titel, 1. Sektion, Art. 11 der Verfassung von 1819; Art. 21 der Verfassung von 1821; zuvor Art. 26 ff der Verfassung von 1811.

Art. 155 der Verfassung von 1811; 1. Titel, 1. Sektion, Art. 12 der Verfassung von 1819: „La propiedad es el derecho de gozar y disponer libremente de sus bienes y del fruto de sus talentos, industria o trabajo“, in: Brewer-Carías, Las Constituciones de Venezuela (Anm. 51), S. 197 bzw. 248.

79 1. Titel, 1. Sektion, Art. 11: „La propiedad es el derecho de gozar y disponer libremente de sus bienes y del fruto de sus talentos, industria o trabajo“, in: Grases, El Libertador y la Constitución de Angostura de 1819 (Anm. 28), S. 114.

John Locke, Zwei Abhandlungen über die Regierung (Two treatises of government, 1690), 7. Aufl., Frankfurt/M. 1998, 2. Abhandl., 5. Kap., § 27 f, S. 216 f; § 32, S. 219 f.

1. Titel, 1. Sektion, Art. 15 der Verfassung von 1819; Art. 177 der Verfassung von 1821; Art. 165 der Verfassung von 1811 hatte als Eingriffsrechtfertigung lediglich die ,öffentliche Notwendigkeit" anerkannt. 
Handels- und Gewerbefreiheit, wie ihn alle drei Verfassungen übereinstimmend vorsahen. $^{82}$

- Die tiefstgreifende Änderung der bolivarischen Konzeption gegenüber der Verfassung von 1811 liegt in der Abkehr vom bundesstaatlichen Prinzip und der Entscheidung für ein unitarisches System ${ }^{83}$. Kennzeichnend für das Territorialregime der ersten venezuelanischen Republik war eine generelle Zuständigkeitsvermutung zugunsten der Bundesstaaten: „Alles, was nicht ausdrücklich durch den Bundesvertrag (pacto federal) in die allgemeine Zuständigkeit der Konföderation verwiesen ist, bleibt den einzelnen Provinzen vorbehalten ... Sie sind souverän, frei und unabhängig“ ${ }^{84}$. Der Konföderation vorbehalten waren die Vertretung Venezuelas nach außen, gemeinsame Verteidigungsanstrengungen, die Regelung des Außenhandels, die Vorsorge gegen innere Unruhen, der Abschluss von Verträgen und Bündnissen mit anderen Nationen sowie die Erhebung von Steuern. Dem Prinzip der Bundestreue gemäß durften die Provinzen weder durch Gesetz noch Regierungsakte diese der Konföderation vorbehaltenen Gegenstände regeln oder auf Bundesebene getroffenen Regelungen zuwider handeln (Art. 119). Der unitarische Charakter des Staatsaufbaus von 1819, wie ihn dagegen Bolívar dem Kongress von Angostura in seinem Verfassungsentwurf empfahl, kommt in einer zentralen Verwaltung und Einteilung des nationalen Territoriums in Provinzen, Departements und Gemeinden zum Ausdruck. ${ }^{85}$

Darin liegt einerseits die Abkehr vom nordamerikanischen Muster, das noch der bundesstaatlichen Verfassung von 1811 zugrunde lag, andererseits die Hinwendung zur Territorialordnung der Französischen Revolution, die Bolívar als die für Venezuela geeignetere ansah. Die Provinzialverwaltung wurde nach Maßgabe der Verfassung von 1819 durch den Gouverneur geführt, der drei Jahre amtierte, zugleich den Gemeinden seiner Provinz vorstand und direkt dem Präsidenten unterstellt war (9. Titel, 1. Sektion, Art. 1 ff). Das der Provinz nachgeordnete Departement wurde durch den Präfekten verwaltet, der an seinem Sitz außerdem als Mitglied des Gemeinderates fungierte (9. Titel, 2. Sektion, Art. 1 f). Die Verfassung von 1821 belebte die im Verlaufe der bourbonischen Reformen eingeführte Institution des Intendanten wieder, um eine zentrale Steuerung und politische Verantwort-

Art. 167 der Verfassung von 1811; 1. Titel, 1. Sektion, Art. 13 der Verfassung von 1819; Art. 178 der Verfassung von 1821.

2. Titel, 1. Sektion, Art. 1 der Verfassung von 1819: „La república de Venezuela es una e indivisible“, in: Brewer-Carías, Las Constituciones de Venezuela (Anm. 51), S. 249.

So die Vorrede (Preliminar) zur Verfassung von 1811, in: Brewer-Carías, Las Constituciones de Venezuela (Anm. 51), S. 181. zusätzlich Kantone ein, die den Provinzen unterstehen, letztere wiederum den Departments als oberste territoriale Kategorie (Art. 8). 
lichkeit der Verwaltung vor Ort zu gewährleisten. Der Intendant wurde durch den Präsidenten auf drei Jahre ernannt und unterstand ihm unmittelbar (Art. $151 \mathrm{f}$ ).

Im Ergebnis vermisste Bolívar auch in den Verfassungen von 1819 und 1821 nicht nur eine noch stärkere Position der Exekutive gegenüber der Legislative in Friedenszeiten, sondern auch eine der Vertretung des englischen Adels tatsächlich entsprechende Kammer und eine weitere, ,moralische Gewalt“, die mit den herkömmlichen Staatsfunktionen konkurrieren konnte. Wenn diese beiden Verfassungen hier mit den „bolivarischen“ Konzepten identifiziert werden, so gilt das aber mit einer wesentlichen Einschränkung: Erst die durch ihn oktroyierten Verfassungstexte von Bolivien und Peru (1826) entsprechen - wenn auch unter anderen politischen Bedingungen - in jeder Hinsicht Bolívars Modell, das er allein als praktikabel für eine Regierung Spanischamerikas ansah ${ }^{86}$. So ergänzt der peruanische Text $^{87}$ die drei klassischen Staatsfunktionen durch eine vierte, die „Wählergewalt“ (Poder Electoral, Art. 9, Art. 20 ff). Das Zweikammersystem, das Bolívar für eine Quelle fortgesetzter Auseinandersetzungen hielt, ersetzte er durch ein Dreikammersystem (Art. 27 ff, Cámara de Tribunos, de Senadores, de Censores). Die Präsidentschaft wurde Bolívar, der nur einmal mit absoluter Mehrheit durch die Legislative zu wählen war, auf Lebenszeit übertragen (Abs. 5 und Art. 1 der Präambel). Eine gewisse Selbstbeschränkung lag andererseits darin, dass sich Bolívar im Rahmen seiner Amtsführung vorrangig mit militärischen und diplomatischen Angelegenheiten sowie mit Haushaltsfragen befasste, die eigentliche Verwaltung aber dem Vizepräsidenten überließ. Dies entsprach nicht nur seinen besonderen Interessen sondern auch der Einsicht, dass die Bedeutung dieser Aufgaben eine Arbeitsteilung erforderlich machte. ${ }^{88}$

\section{Bezugspunkte der, ,bolivarischen“ Verfassung 1999}

Wenn man also - trotz aller methodischen Vorbehalte - im aktuellen, von Hugo Chávez dominierten Staats- und Verfassungskonzept nach Bezugspunkten sucht, die auf eine bolivarische Tradition hindeuten, lassen sich einige, im folgenden genannte Elemente nennen. Maßstab seien hier zum einen die von Chávez der Konstituante am 5. August 1999 vorgelegten ,grundlegenden Vorstellungen für eine bolivarische Verfassung der fünften Repu-

Manuel Lucena Salmoral, Simón Bolívar, Madrid 1991, S. 109.

87

Constitución para la República Peruana (denominada Constitución Vitalicia de Bolívar) vom 30.11.1826, in: Cámara de Diputados (Hg.), Constituciones Políticas del Perú 1821-1919, Lima 1922, S. $73 \mathrm{ff}$.

88

Lucena Salmoral, Simón Bolívar (Anm. 86), S. 13, 110. 
blik““89. Zu diesem Zeitpunkt nahm die am 25. Juli 1999 gewählte Verfassunggebende Nationalversammlung (Asamblea Nacional Constituyente) ihre Arbeiten auf. Die Anhänger des Präsidenten stellten darin die überwältigende Mehrheit der Abgeordneten ${ }^{90}$. Zum anderen ist als maßgebliche rechtliche Quelle die schließlich verabschiedete „Verfassung der Bolivarischen Republik“ von Dezember 1999 heranzuziehen, die auf seine ,grundlegenden Vorstellungen“ zurückgeht. Schließlich werden Stellungnahmen berücksichtigt, in denen Chávez auf verfassungsrechtliche und gesellschaftspolitische Fragen eingeht.

- Kennzeichen einer jeden bürgerlich-liberalen Verfassungsordnung seit den beiden wichtigsten geschichtlichen Vorläufern, der nordamerikanischen Verfassung von 1787 und der französischen Verfassung von 1791, ist die Gewaltenbalancierung der legislativen und der exekutiven Befugnisse. Dagegen verfolgen sowohl der Verfassungsentwurf nach den „grundlegenden Vorstellungen“ Chávez' wie auch der schließlich verabschiedete Verfassungstext von 1999 das Ziel, dieses Balancesystem zugunsten des Präsidenten zu verändern. Dieser gilt als „Haupt der Nation“, verkörpert die „nationale Einheit“ und wird als Garant von „Freiheit, Unabhängigkeit, Souveränität“ u.a. angesehen ${ }^{91}$. Zum umfassenden Katalog seiner Kompetenzen als Staats- und Regierungschef gehören nicht nur die Führung der Verwaltung, sondern auch der Oberbefehl über die Streitkräfte, die Ausrufung des Staatsnotstandes, eine gewisse Teilhabe an der Gesetzgebung durch seine spezifische Verordnungsgewalt, in bestimmten Fällen die Auflösung der Nationalversammlung und deren Einberufung zu außerordentlichen Sitzungen (vgl. i.E. den Katalog in Art. 236 der Verfassung). Diese Funktionen gehen über die in der „Verfassung der Republik Venezuela“ von 1961 geltenden Befugnisse des Präsidenten hinaus ${ }^{92}$. Dies gilt auch für die Amtszeit des Präsidenten, die sich von fünf Jahren auf sechs Jahre verlängert und eine unmittelbare Wiederwahl erlaubt (Art. 230). Diese Begrenzung auf nur eine direkte Wiederwahl hebt sich zwar von den weiter reichenden Bestrebungen Simón Bolívars im Zuge der staatlichen

Im folgenden nach der englisch-sprachigen Fassung: Commander Hugo Chávez Frías, President of The Republic of Venezuela To the Sovereign National Constitutional Assembly, Fundamental Ideas for the Bolivarian Constitution of the V Republic, Caracas 1999.

Monleón, in: Verfassung und Recht in Übersee 37 (2004) (Anm. 4), S. 66 und Anm. 34, sowie Mariela Morales, Venezuela auf dem Weg zum Bundesstaat?, in: Zeitschrift für ausländisches öffentliches Recht und Völkerrecht 2004, S. 169, zählen 125 Sitze von insgesamt 131 für die Anhänger des Präsidenten; andere Angaben kommen auf 121 von 131 Sitzen.

So im 3. Kapitel („The National Executive Branch“) der ,grundlegenden Vorstellungen für eine bolivarische Verfassung“, in: Chávez (Anm. 89), S. 65: „The President of the Republic is the head of the Nation, expresses the national unity and is bound to guarantee the rights and freedoms of the Venezuelan people, and the independence, integrity, territorial sovereignty and defense of the Republic". 
Emanzipation der Andenstaaten ab, eine möglichst lange, ununterbrochene Amtszeit des Präsidenten zu erreichen, so durch Verankerung der bereits erwähnten Präsidentschaft auf Lebenszeit (presidencia vitalicia). Andererseits zielt die Regelung dennoch auf eine Befestigung der exekutiven Stellung ab. Dies gilt zum einen im Verhältnis zur vorhergehenden Verfassung von 1961, die eine Wiederwahl des Präsidenten während eines Zeitraums von zehn Jahren nach Ablauf der Amtszeit ausschloss (Art. 185). Dies gilt aber auch im Vergleich zu den restriktiveren Bestimmungen anderer Präsidialsysteme, etwa im benachbarten Kolumbien oder in den Vereinigten Staaten. ${ }^{93}$

- Ein zweiter für die Gewaltenbalancierung sehr wesentlicher Aspekt bedeutet in nahezu allen lateinamerikanischen Staaten seit den ersten Emanzipationsbestrebungen die umstrittene Frage, welches territoriale Regime gelten solle. Die Lösungen in Venezuela schwankten von Anfang an zwischen einer bundesstaatlichen Ordnung nach dem Vorbild der ersten Verfassung von 1811, einem unitarischen System mit dezentraler Organisation sowie einem zentralistischen Einheitsstaat. Trotz der Vorliebe des Staatengründers Bolívar für eine unitarische Lösung, die er in den Verfassungen von 1819, 1821 und 1826 durchsetzte, hat sich im 20. Jahrhundert die bundesstaatliche Lösung behauptet, die auch die neue Verfassung von 1999 formal bestätigt ${ }^{94}$. Dennoch erscheint das föderale Prinzip nunmehr so widersprüchlich ausgestaltet, dass in der Literatur die Frage gestellt wird, ob es überhaupt funktionsfähig sei ${ }^{95}$. Einerseits sprechen das Bekenntnis zum Bundesstaat (Art. 4) und die daraus folgende Staatlichkeit auf mehreren Ebenen (Art. 136) für eine wirkliche vertikale Gewaltenteilung. Andererseits besitzen die Gliedstaaten nur formal politische Autonomie und fehlt das Verfassungsgebot, dass sie an der Willensbildung des Gesamtstaates mitwirken $^{96}$. Schon die besondere Betonung des „,dezentralen Charakters“ dieses föderalen Systems ist terminologisch unklar, weil eine solche Dezentralisierung auch zur Kennzeichnung eines unitarischen Staatenbaus dienen kann und die neue „bolivarische“ Verfassung

Die bis heute geltende Verfassung der USA von 1787 sieht eine vierjährige Amtszeit vor (Art. II, Abschn. 1 Abs. 1), mit der Option nur einer weiteren Amtsperiode vor. Die kolumbianische Verfassung von 1991 legte bisher eine vierjährige Amtszeit ohne die Möglichkeit der Wiederwahl fest (Art. 190 Abs. 1, 197 Abs. 1); der Kongress hat jedoch am 2. Dezember 2004 eine Verfassungsänderung beschlossen, die zum ersten Mal im Jahr 2006 die (einmalige) Wiederwahl des Präsidenten im direkten Anschluss an eine Amtsperiode ermöglicht.

Art. 4: „La República Bolivariana de Venezuela es un Estado federal descentralizado“, in: Verfassung (Anm. 7), S. 1; zuvor bereits Art. 2 der Verfassung von 1961: „La República de Venezuela es un estado federal“, in: López Guerra/Aguiar de Luque, Las Constituciones de Iberoamérica (Anm. 92), S. 950.

So Morales, in: Zeitschrift für ausländisches öffentliches Recht und Völkerrecht 2004 (Anm. 90), S. 164.

96 Morales, in: Zeitschrift für ausländisches öffentliches Recht und Völkerrecht 2004 (Anm. 90), S. 171, die auf den Unterschied zu Bundesstaaten wie USA, Kanada und Deutschland hinweist. 
tatsächlich Merkmale eines solchen dezentralen Einheitsstaates aufweist. So sieht Art. 4 eine nicht unerhebliche Einschränkung des bundesstaatlichen Prinzips vor: Die Änderung des Zweikammersystems zugunsten einer einzigen Volkskammer vermindert den Einfluss der Bundesstaaten, denen nun eine Vertretungsstufe auf Bundesebene fehlt und damit eine wichtige Möglichkeit, auf die Gesetzgebung Einfluss zu nehmen. Die Verfassung schreibt lediglich eine Anhörung der Landesparlamente vor, soweit Bundesgesetze ihre Interessen berühren (Art. 206); außerdem die Teilnahme eines Landesvertreters an den Lesungen in der Nationalversammlung (Art. 211 S. 2). Im Sinne eines gewissen Ausgleich wurde ein föderaler Regierungsrat (Consejo Federal de Gobierno) geschaffen, der aber eine nur beratende Funktion wahrnimmt. ${ }^{97}$

- Eine der hervorstechenden Neuerungen der neuen Verfassung von 1999 ist die Vermehrung der Staatsfunktionen (oder Gewalten). Das 4. Kapitel des 5. Verfassungstitels („De la organización del poder público nacional“) verankert in Art. $273 \mathrm{ff}$ die Institution einer „Bürgergewalt“ (Poder Ciudadano). Die Bürgergewalt wird durch einen institutionell unabhängigen „Republikanischen Moralrat“ (Consejo Moral Republicano) ausgeübt, der aus dem Ombudsmann, dem Oberstaatsanwalt und dem Obersten Rechnungsprüfer besteht (Art. 273). Er überwacht die gesamte öffentliche Verwaltung auf mögliche Verstöße gegen die „öffentliche Ethik“ und „Verwaltungsmoral“, die er untersuchen und ahnden soll (Art. 274). Außerdem regelt das 5. Kapitel (Art. 292 ff) die „Wählergewalt“ (Poder Electoral), die ein Nationaler Wahlrat (Consejo Nacional Electoral) ausübt (Art. 292). Dieses aus fünf Gremien bestehende Wahlaufsichtsorgan hat u.a. die Aufgaben, Wahlen zu repräsentativen Versammlungen und zu berufsständischen oder gewerkschaftlichen Einrichtungen in ihrem Ablauf zu regeln und zu überwachen, das Zivil- und das Wahlregister zu führen und die Finanzierung politischer Organisationen zu regeln und zu überwachen (vgl. im einzelnen den Katalog in Art. 293). Von allen in der Verfassung enthaltenen Rechtsfiguren geben diese beiden neuen Staatsfunktionen den deutlichsten Hinweis auf die Bemühungen, an Simón Bolívar anzuknüpfen, der zusätzliche Staatsfunktionen dieser Art stets propagiert und in Bolivien wie in Peru (1826) auch oktroyiert hatte.

- Vom Staatsdenken Bolívars, wie es gerade in dem von ihm favorisierten Wahlsystem zum Ausdruck kommt, unterscheidet sich die große Bedeutung des Plebiszits im politischen Konzept Hugo Chávez': So ordnete er nach seiner Wahl zum Präsidenten im Februar 1999 die Durchführung eines Referendums an, um die Bevölkerung zur Einberufung einer verfassunggebenden Versammlung zu befragen ${ }^{98}$. Folgerichtig betont die neue, durch Referendum angenommene Verfassung ihre plebiszitären Elemente mit besonderem Nach- 
druck. Schon die Einordnung dieses 4. Kapitels („De los Derechos Políticos y del Referendo Popular“) in den 3. Verfassungstitel („De los Derechos Humanos y Garantías, y de los Deberes“), der von den Menschenrechten und von den individualrechtlichen Garantien der Venezuelaner handelt, verrät den hohen Stellenwert des Referendums. Das „Referendo Popular" (Art. 71 ff) gehört nunmehr neben dem Wahlrecht (Art. 63) zum Kernbestand einer politischen Teilhabe der Bürger und erhält den Rang einer subjektiv-rechtlichen Gewährleistung. Diese Bedeutung spiegeln auch die weitreichenden inhaltlichen Vorgaben der Verfassung wider, die auf Chávez' „grundlegenden Vorstellungen für eine bolivarische Verfassung“ zurückgehen ${ }^{99}$ : Allgemeine Voraussetzung für die Einleitung eines Referendums ist, dass es sich um einen Belang ,besonderer nationaler Bedeutung“ handelt. Die Einleitung erfolgt auf Initiative des Präsidenten, der Nationalversammlung oder auf Verlangen von mindestens einem Zehntel der in das Wahlregister eingetragenen Bürger (Art. 71 Abs. 1). Diese Möglichkeit besteht nicht nur auf nationaler Ebene, sondern entsprechend auch für Belange der Land- und Stadtgemeinden sowie der (Teil-)Staaten Venezuelas (Art. 71 Abs. 2).

Eine spezielle Anwendung des Referendums sieht die Verfassung vor allem für zwei Bereiche vor, einen personellen und einen legislativen: Zum einen besteht die Möglichkeit der Amtsenthebung aller öffentlichen Funktionsträger, die ihr Mandat einer Wahl durch die Bürger verdanken. Als besondere Bedingungen müssen die Hälfte der Amtszeit verstrichen sein und mindestens ein Fünftel der im Wahlregister eingetragenen bürger die Abstimmung verlangen (Art. 72 Abs. 1). Die Abwahl ist erfolgreich, wenn mindestens die gleiche Anzahl derjenigen, die den jeweiligen Amtsträger zuvor gewählt hatten, für seine Amtsenthebung stimmen und minstens ein Viertel der in das Wahlregister Eingetragenen am Referendum teilgenommen haben (Art. 72 Abs. 2) ${ }^{100}$. Zum anderen ermöglicht Art. 73 eine Entscheidung des Volkes über Gesetzesvorhaben, die noch in der Nationalversammlung debattiert werden, und über internationale Verträge. Außerdem können auch bereits verabschiedete Gesetze und bestimmte Dekrete des Präsidenten mit Gesetzeskraft Gegenstand einer Abstimmung des Volkes sein (Art: 74).

- Eine jüngere, erst das 20. Jahrhundert beherrschende Entwicklung betrifft die verfassungsrechtliche Verankerung sozialstaatlicher Gewährleistungen. Der liberalen Generation der spanischamerikanischen Staatengründer um Simón Bolívar waren solche Ansätze

Chávez, Fundamental Ideas for the Bolivarian Constitution of the V Republic (Anm. 89), 4. Titel,

2. Kap. (,The Referendum“), S. 49 f.

100

Dies sind die Voraussetzungen, die auch das (gescheiterte) Referendum vom 15. August 2004 zur Abwahl des Staatspräsidenten Hugo Chávez hätte erfüllen müssen. In der Verfassung nicht geregelt und umstritten ist die Frage, ob ein soeben abgewählter Amtsinhabe sich sogleich wieder zur Wahl stellen darf. 
fremd. Als fortschrittlich galten zu Beginn des 19. Jahrhunderts die Wirtschafts- und Gesellschaftskonzepte der französischen Physiokraten und der angelsächsischen Theoretiker um Adam Smith. Sie beeinflussten die venezuelanischen Kreolen entweder direkt über die Lektüre der französischen und englischen Texte oder vermittelt durch spanische Aufklärer wie Pedro Rodríguez Conde de Campomanes (1723-1802) und Gaspar Melchor de Jovellanos y Ramírez (1744-1811), deren Wirtschafts- und Eigentumslehren in der zweiten Hälfte des 18. Jahrhunderts über zahlreiche Reformentwürfe für die Gesetzgebung der spanischen Könige, Karl III. (1759-1788) und Karl IV. (1788-1808), sowie über maßgebliche Denkschriften in der gesamten spanischen Monarchie bekannt wurden ${ }^{101}$. Alternative Modelle kamen angesichts der Desavouierung der merkantilistischen wirtschafts- und ständestaatlichen Gesellschaftsordnung nicht in Betracht, einmal abgesehen von einzelnen, zumeist religiös motivierten Modellversuchen in isolierten christlichen Gemeinschaften und wenigen radikalen Theoretikern der Französischen Revolution, die bereits sozialistische Ideen propagierten.

Dagegen messen Hugo Chávez und die neue Verfassung von 1999 sozialstaatlichen Verbürgungen erhebliche Bedeutung bei. Sie nehmen ihren Ausgang in Chávez' Verfassungsentwurf, der zunächst nur einige Eckpunkte für das viel ausführlichere 5. Kapitel des 3. Verfassungstitels („De los derechos sociales y de las familias“) festlegte ${ }^{102}$. Außerdem soll die Verfassung offenbar einen Teil zur Umsetzung des recht ambitionierten sozialpolitischen Abschnitts des Regierungsprogramms des Präsidenten beitragen ${ }^{103}$. In der neuen Verfassung spiegelt sich eine Tendenz der jüngeren südamerikanischen Verfassungen wider, die einen weiten Fächer individueller und kollektiver Gewährleistungen, Staatszielbestimmungen und Eingriffsvorbehalte zugunsten der öffentlichen Gewalten verankern. Im Mittelpunkt stehen die Sicherung der existentiellen Voraussetzungen Arbeit, Wohnung, Bildung, Gesundheit der Bevölkerung, aber auch neuere Belange wie der Umweltschutz ${ }^{104}$. Leitbegriff einer solchen Daseinsvorsorge in Venezuela ist die „Gerechtigkeit als republikanische Tugend“. Ihn versteht Chávez in einem gesellschaftspolitischen, wenn nicht klassenkämpferischen Sinne. Dagegen tritt der rechtsstaatliche Aspekt, den die liberale Staa-

Näher Jean Sarrailh, La España ilustrada de la segunda mitad del siglo XVIII, Mexiko 1979, S. $281 \mathrm{ff}, 546 \mathrm{ff}$.

102 Dies betrifft u.a. das „,Recht auf Soziale Sicherheit“, ,,auf eine angemessene Unterkunft“, ,,auf eine gesunde Umwelt", Chávez, Fundamental Ideas for the Bolivarian Constitution of the V Republic (Anm. 89), 2. Titel, 6. Kap., S. 32 f.

103 3. Abschnitt des Programms: „Equilibrio Social: Alcancemos la Justicia Social“ (Anm. 6), S. 14 ff. Die Gewährleistung der Bildung (S. 16 ff) und Gesundheit (S. 31 ff) nehmen hierin den größ104 ten Raum ein.

Für die kolumbianische Verfassung von 1991: Andreas Timmermann, Soziale Funktion und Umweltfunktion des Eigentums in der spanischen und in der kolumbianischen Verfassung, Baden-Baden 1996, S. 163 ff, 207 ff. 
tengründergeneration und Simón Bolívar mit ,justicia“ verbanden, bei Chávez deutlich in den Hintergrund. ${ }^{105}$

Die Verfassung verankert ferner eine Garantie „,befriedigender Arbeitsbedingungen“ (Art. 81 S. 2), das „Recht jeder Person auf eine angemessene, sichere, bequeme, hygienische Unterkunft mit der wesentlichen Grundversorgung" (Art. 82 Abs. 1) und die Gesundheit zugleich als Verpflichtung des Staates und als „gesellschaftliches Grundrecht“, weshalb dieses als Bestandteil des Rechts auf Leben garantiert wird (Art. 83 S. 1). Das in der Verfassung verbürgte „Recht auf soziale Sicherheit als öffentliche Dienstleistung“ (Art. 86 S.1) soll - zumindest dem Anspruch des Verfassungsgebers folgend - nahezu alle Lebensrisiken absichern: u.a. Krankheit, Invalidität, Katastrophen, Risiken am Arbeitsplatz und Arbeitsverlust, Verlust des Ehepartners oder der Eltern, weshalb eine „solidarisch finanzierte, effiziente, partizipative, universale Sozialversicherung" garantiert wird (Art. 86 S. 2).

Den ganzen Wortreichtum der neuen Verfassung im allgemeinen und der sozialstaatlichen Garantien im besonderen belegt der Umstand, dass alleine zwölf Artikel (Art. 81, Art. 8797) sich auf den Lebensbereich der Arbeit beziehen. Hugo Chávez räumt diesem Lebensbereich und - in Andeutung des Klassengegensatzes - den „Rechten der Arbeiter“ (los derechos de los trabajadores) eine Schlüsselstellung innerhalb der Verfassung ein ${ }^{106}$. Dies impliziert zum einen das Recht auf Arbeit wie die Pflicht hierzu (Art. 87 S. 1). Dies schließt zum anderen die Gewährleistung notwendiger hoheitlicher Maßnahmen ein, damit jede Person eine ,produktive Beschäftigung“ erhalte, die ihm eine würdige Existenz sichert (Art. 87 S. 2). Fast ebenso ausführlich behandelt die Verfassung einen weiteren Schwerpunkt der Agenda Chávez', nämlich das Recht auf Bildung (Art. 102-110). Es wird nicht nur als Bürger-, sondern sogar als Menschenrecht formuliert (Art. $102 \mathrm{~S}$. 1) und umfasst neben anderen neueren Inhalten auch eine Pflicht des Staates zur Umwelterziehung auf allen Bildungsstufen (Art. 107 S. 1). Hierin geht die neue Verfassung deutlich über die Verfassung von 1961 hinaus, die allerdings bereits, wenn auch in einem reduzierten Umfang und mit einer geringeren Reichweite, die Rechte der Bürger auf den Schutz der Gesundheit (Art. 76 Abs. 1), auf Bildung (Art. 78 Abs. 1) und auf Arbeit (Art. 84) proklamiert hatte. ${ }^{107}$

105 Mit Bezug auf Bildung und Gesundheitsfürsorge: Hugo Chávez, Mensaje Anual a la Asamblea Nacional, Caracas am 17.1.2003, unter: www.venezuela-oas.org/Annual message to the National Assembly.pdf, S. 18 f; gegen die „Oligarchie“ gerichtet und bzgl. des „Rechts auf Bildung“: Hugo Chávez, Discurso Acto de entrega de Tierras Urbanas, Caracas am 11.1.2003, unter: www.venezuela-oas.org/Speech at the Urban Land Titling Ceremony.pdf, S. $1 \mathrm{f}$.

Chávez, Rueda de Prensa del Presidente (Anm. 24).

Systematisch wurden diese Rechte in die Kategorie der „Sozialen Rechte“ (Derechos sociales) eingeordnet und den „Wirtschaftsrechten“ (u.a. Gewerbefreiheit, Eigentumsfreiheit) gegenüber gestellt - eine Systematik, die sich u.a. in der spanischen Verfassung von 1978 findet. 
- Wenn festgestellt wurde, dass es für plebiszitäre Elemente und sozialstaatliche Verbürgungen keine Anknüpfungspunkte im Verfassungsdenken Bolívars gab, so gilt dies erst recht für die Eigentumskonzeption Hugo Chávez'. Im Sinne einer gerechteren Eigentumsstruktur betont er die Notwendigkeit einer staatlichen Landverteilung, um eine Entwicklung rückgängig zu machen, in deren Verlauf ,eine Minderheit täglich reicher und reicher geworden ist, während die große Mehrheit ihrem Schicksal überlassen blieb“"108. Diese Zielsetzung, eine Revision öffentlichen und privaten Landeigentums zu beschleunigen, verfolgt etwa die im Jahr 2001 vorgenommene Neudefinierung brachliegender Flächen, die ab 5000 Hektar als nicht mehr zulässiger „Großgrundbesitz“ gelten.

Die Verfassung knüpft im 7. Kapitel des 3. Verfassungstitels (,De los Derechos Económi$\cos ^{\text {") }}$ zunächst an die klassische Dreiteilung an und unterscheidet die eigentliche individualrechtliche Garantie des Privateigentums als Recht auf Nutzung und Verfügung (Art. 115 S. 1) von den Beschränkungen aus Gründen des Allgemeinwohls und der öffentlichen Nützlichkeit (S. 3) sowie der Enteignung, die nur gegen Entschädigung zulässig ist (S. 4). Diese Formel lässt sich lediglich in Bezug auf die Privateigentums- und Entschädigungsgarantie auf die ersten venezuelanischen Verfassungstexte von 1811 bis 1821 zurückführen, die das klassische liberalstaatliche Konzept vom Eigentum als Abwehrrecht abbildeten. Dieses Konzept stellte den Ausgangspunkt für die weitere Entwicklung des Eigentumsschutzes in Lateinamerika dar, wo sich besonders ausgeprägt die Ergänzung dieser ,ersten Generation“ des Eigentumsrechtes durch eine zweite, dem Solidarprinzip, und eine dritte, dem Sozialstaatsprinzip, verpflichtete Kodifikationsstufe beobachten lässt ${ }^{109}$. Diese letzten beiden Entwicklungsstufen des 20. Jahrhunderts lagen - wie dies mit Blick auf das Sozialstaatsprinzip schon festegestellt wurde - dem Denken jener liberalen Generation der Staatengründer um Simón Bolívar noch fern.

Sie grenzt sich im venezuelanischen Fall aber auch von den liberaleren Entwürfen der Gegenwart ab. In diesem Sinne stellt Chávez die Nationalisierung der Schlüsselindustrien in den Mittelpunkt seiner Wirtschaftspolitik, um die angeblichen Folgen liberaler Politik zu korrigieren $^{110}$. Die Verfassung von 1999 sieht in Ergänzung zu Art. 115 zahlreiche Interventionsmöglichkeiten vor, etwa zum Schutz der Verbraucher vor Monopolen oder vor einem Missbrauch des (Unternehmens-)Eigentums (Art. 113 Abs. 1). Hinzu kommen Eingriffsvorbehalte zum Schutz der Umwelt (Art. 127-129). Dem Staat übertragen werden die

108

109

Chávez, Discurso Acto de entrega de Tierras Urbanas (Anm. 105), S. $3 \mathrm{f}$.

Dazu Andreas Timmermann, Die Eigentumsgarantie - ein stufenförmiger historischer Prozess? Das „Generationenrecht“ in den kolumbianischen Verfassungen (1812-1991), in: Jahrbuch für 110 Geschichte Lateinamerikas 38 (2001), S. 272 ff.

So mit Bezug auf den Energiesektor: Chávez, Mensaje Anual a la Asamblea Nacional (Anm. 105), S. 19. 
Ausbeutung der natürlichen Ressourcen, die im Eigentum der Nation stehen, und die Erbringung solcher Dienstleistungen, die ,ihrer Natur nach öffentlich“ seien (Art. 113 Abs. 2). In diesen Zusammenhang gehört auch die starke Betonung der Gleichheit in der Verwendung des Eigentums gegenüber der individuellen Freiheit in Nutzung und Verfügung. Ausdruck dessen ist die Zugangsgarantie aller Personen zu Gütern und Dienstleistungen, deren Umsetzung den öffentlichen Gewalten überlassen bleibt (Art. 117 S. 1 und S. 2).

Im Ergebnis verbinden sich die protektionistischen Tendenzen im Wirtschafts- und Gesellschaftskonzept Hugo Chávez', das er nach der Theorie Norberto Ceresoles dem sogenannten neuen Liberalismus entgegensetzt, mit dem älteren ,Solidarismus“, der seit den dreißiger Jahren des 20. Jahrhunderts eine erhebliche Ausweitung öffentlicher Dienstleistungen befördert hat ${ }^{111}$. Von dieser Synthese zeugt nicht zuletzt der große Raum, den der 4. Titel der Verfassung von 1999 (,Del Poder Público“) und hierin wiederum die 3. Sektion über die öffentlichen Dienste („De la Función Pública“, Art. 144-149) innerhalb des Verfassungstextes einnehmen.

\section{Ergebnis}

Im Ergebnis lassen sich Parallelen zwischen dem Staatsdenken Hugo Chávez' und jenem Simón Bolívars nur schwer ziehen. Zunächst verfügen weder Chávez noch seine „,bolivarische Bewegung“ über ein klares weltanschauliches Konzept, das ausreichend Kontur besäße, um es einem genaueren Vergleich zu unterziehen. Dies liegt nicht zuletzt an seiner historisierenden Methode, eine Vielzahl geschichtlicher und weltanschaulicher Vorbilder $\mathrm{zu}$ bemühen, die untereinander nur wenige Gemeinsamkeiten aufweisen und auf eine gewisse Beliebigkeit seiner Konzeption schließen lassen. Aber auch das Staatsdenken Simón Bolívars ist nur schwer auf einen für den Vergleich tauglichen Nenner zu bringen. Es ist ein Spiegelbild nicht nur der höchst unterschiedlichen weltanschaulichen Einflüsse, die den jungen Bolívar prägten, sondern auch der politischen Wechselfälle im Emanzipationsprozess der Andenstaaten. Insofern steht sein Staatsdenken stellvertretend für einen in ganz Lateinamerika bis heute verbreiteten Eklektizismus: Nicht ganze Lehrgebäude werden übernommen, sondern recht beliebig einzelne Teilaspekte aus sehr unterschiedlichen Quellen, die die politischen Forderungen zu untermauern geeignet scheinen. In dieser Hinsicht steht Hugo Chávez wie viele vor ihm tatsächlich in der Tradition Bolívars.

111

Näher Andreas Timmermann, „Soziale Solidarität“ und Agrarreform im 20. Jahrhundert: zur Wirkung der Rechtsschule León Duguits, in: Verfassung und Recht in Übersee 37 (2004), S. 171 ff. 
Auch dort, wo sich Chávez in seinem Verfassungskonzept auf Rechtsfiguren der bolivarischen Verfassungstexte bezieht, sind diese allein wegen der völlig anderen Auffassung von Staat und Gesellschaft kaum zu vergleichen. Einerseits erinnern zwar die neuen Staatsfunktionen der Verfassung von 1999, „Bürgergewalt“ (Art. 273 ff) und „Wählergewalt“ (Art. 292 ff), an das Konzept Bolívars, mit dem dieser durch neue Institutionen und eine „vierte“ Gewalt die klassischen drei Staatsfunktionen auszubalancieren suchte. Auch die Stärkung der Exekutive, wie sie Chávez verfolgt, und die Stärkung der zentralstaatlichen Kompetenzen in der neuen Verfassung erinnern an Bolívar, auch wenn letzterer sich konsequenterweise ganz vom bundesstaatlichen Modell des Jahres 1811 gelöst hatte. Andererseits scheinen Welt- und Menschenbild beider Präsidenten wenig kompatibel, was sich in den hier verglichenen Verfassungen niederschlägt und die Zeitgebundenheit beider Konzeptionen unterstreicht: Chávez' Lösung ist egalitärer und plebiszitärer, was unvereinbar mit der liberalen, elitären Auffassung Simón Bolívars ist. Dies beweist die völlig andere Regelung des Wahlrechts, da Bolívar noch kein allgemeines, sondern ein an Leistung und Vermögen orientiertes gestuftes Wahlrecht vertrat. Ebenso fern standen seinem Denken sozialstaatliche Verbürgungen oder umfassende Eingriffsvorbehalte in Eigentums- und Gewerbefreiheit. Während Chávez' Konzeption einer Umverteilung des privaten Vermögens, dem wirtschaftlichen Protektionismus und nationalstaatlichen Monopolen Raum gibt, stellte Bolívar - hierin wiederum sehr zeitgemäß - keinesfalls den Freihandel oder die Eigentumsfreiheit in Frage. Denn nicht zuletzt für diese ökonomischen Belange hatte die kreolische Elite in Übersee den Unabhängigkeitskampf gegen die spanische Monarchie geführt. Und gerade auf die solchermaßen befestigten wirtschaftlichen Privilegien der venezuelanischen Oberschicht geht aus Sicht der Anhänger einer „bolivarischen Bewegung“ jenes ,,ancien régime“ zurück, dessen Überwindung Hugo Chávez nun proklamiert.

Schließlich unterscheiden sich auch die politischen Äußerungen Chávez' in der Öffentlichkeit allzu deutlich von den Bemühungen Simón Bolívars, jede Form des Klassenkampfes, sei es ,,von oben“ oder ,,von unten“, zu vermeiden. Auch wenn man die teilweise aggressive Polemik, so gegen das ,räuberische“, ,unpatriotische“, „neoliberale“ und „faschistische“ Großbürgertum ${ }^{112}$, nicht mit Chávez’ Verfassungskonzept gleichsetzen kann, so kommen darin doch ein grundlegend anderes Gesellschaftsbild und starke Ressentiments zum Ausdruck, die Bolívar fremd waren. 